\title{
TITLE:
}

\section{Electrically driven deformations of nematic gels}

\section{$\operatorname{AUTHOR(S):~}$}

Urayama, K; Kondo, H; Arai, YO; Takigawa, T

\section{CITATION:}

Urayama, K ... [et al]. Electrically driven deformations of nematic gels. PHYSICAL REVIEW E 2005, 71(5): 051713.

ISSUE DATE:

2005-05

URL:

http://hdl.handle.net/2433/39912

RIGHT:

Copyright 2005 American Physical Society 
PHYSICAL REVIEW E 71, 051713 (2005)

\title{
Electrically driven deformations of nematic gels
}

\author{
Kenji Urayama,* Hidesato Kondo, Yuko O. Arai, and Toshikazu Takigawa \\ Department of Material Chemistry, Kyoto University, Kyoto 615-8510, Japan
}

(Received 15 January 2005; published 19 May 2005)

\begin{abstract}
The electrically driven deformations of side chain nematic networks swollen by nematic solvents (nematic gels) have been investigated. The strains of freely suspended gels between electrodes were measured as a function of field strength $(E)$. The deformation of the gels composed of a network and solvent with identical signs of dielectric anisotropy $(\Delta \varepsilon)$ is dominated by the electrically induced alignment of the nematogens. As a result, the stretching direction is variable according to the sign of $\Delta \varepsilon$ : The gel with positive or negative $\Delta \varepsilon$ is elongated parallel or normal to the field axis, respectively. The maximum strain among the samples examined is as large as $20 \%$ at $E \approx 0.5 \mathrm{MV} / \mathrm{m}$. The gels composed of a network and solvent with opposite signs of $\Delta \varepsilon$ are compressed along the field axis since the electrostrictive effect becomes dominant because of a large reduction in the mesogen alignment effect due to the discord in the director directions of the constituent nematogens. The gels in the isotropic phase show compressive strains along the field direction in proportion to $E^{2}$ purely originating from electrostriction, independently of the sign of $\Delta \varepsilon$. The nematic gels are quickly deformed within a second upon field application, while the shape recovery after field removal requires a finite time on the order of $10^{3} \mathrm{~s}$, which reflects the structural relaxation in the polydomain texture from the oriented to the random state. The influences of elastic modulus as well as network nematicity on the electrical deformation are also examined.
\end{abstract}

DOI: 10.1103/PhysRevE.71.051713

PACS number(s): 61.30.Vx, 83.80.Va, 82.70.Gg

\section{INTRODUCTION}

The unique feature of nematic polymer networks exhibiting the properties of both polymer networks and liquid crystals has received much attention from the viewpoint of academic interest as well as their potential applications $[1,2]$. The hybrid character of nematic networks yields some interesting phenomena such as spontaneous shape change [3-5] and volume phase transition in solvents [6-10] at the nematic-isotropic $(N-I)$ transition. The macroscopic deformation caused by electrically induced alignment of the constituent mesogens is also an interesting behavior of nematic networks. Electrically driven actuation in nematic networks is expected to be a promising device for artificial muscles and microscopic pumps and valves, because it is much faster due to the direct coupling of the molecular alignment to external fields than the electrical deformation based on ionic diffusion in polymer networks [11].

The electrical distortion of nematic networks swollen by nematic solvents (nematic gels), first observed by Zentel [12], has been investigated by several reseachers [13-17]. Markedly high electric fields are needed to deform appreciably dry nematic networks (nematic rubbers) because of the strong resistance stemming from the high elastic modulus, excepting some nematic rubbers with ferroelectricity [18] and filled with aligned carbon nanotubes [19]. The reduction in elastic modulus on swelling realizes a finite deformation of nematic networks under moderate electric fields.

The electrical deformation of conventional paraelectric materials without global polarization is dominated by elec-

\footnotetext{
*Corresponding author. Electronic address: urayama@rheogate.polym.kyoto-u.ac.jp
}

trostriction [20-22]. In the case of nematic gels, the strain induced by electrical alignment of the constituent nematogens can exceed the electrostrictive strain because of the strong nematic interaction as well as a large dielectric anisotropy $(\Delta \varepsilon), \Delta \varepsilon=\varepsilon_{\|}-\varepsilon_{\perp}$, where $\varepsilon_{\|}$and $\varepsilon_{\perp}$ are the static dielectric constants along $(\|)$ and normal $(\perp)$ to the long axis of the molecules (director), respectively. In principle, the sign of $\Delta \varepsilon$ governs the correlation between the directions of the electric field and the induced director orientation: The director of nematogens with positive or negative $\Delta \varepsilon$ aligns parallel or perpendicular to the field direction, respectively $[23,24]$. Thus the sign of $\Delta \varepsilon$ is an important molecular characteristic of nematogens in the applications of liquid crystal (LC) displays. The sign of $\Delta \varepsilon$ for the constituent nematogens (i.e., the mesogen on the network and the nematic solvent) in nematic gels is also expected to play an important role in the electrical deformation. Most earlier studies $[12,13,15,16]$ used combinations of the nematic networks and solvents both of which have positive $\Delta \varepsilon$, while Huang et al. [17] employed networks with negative $\Delta \varepsilon$ swollen by a solvent with positive $\Delta \varepsilon$. The role of the dielectric anisotropy of the constituent nematogens in electrical deformations, however, still remains unclear because there is no study varying systematically the signs of $\Delta \varepsilon$ for the networks and swelling solvents.

In the present paper, we elucidate the role of dielectric anisotropy for each constituent nematogen in the electrical deformation of nematic gels by employing four combinations of the networks and solvents with positive and negative $\Delta \varepsilon$. We also reveal the difference in electrical deformation behavior between the nematic and isotropic phases, and clarify the effect of nematic order of the mesogenic molecules. The influences of network nematicity as well as network modulus on electrical deformations are studied using networks with 


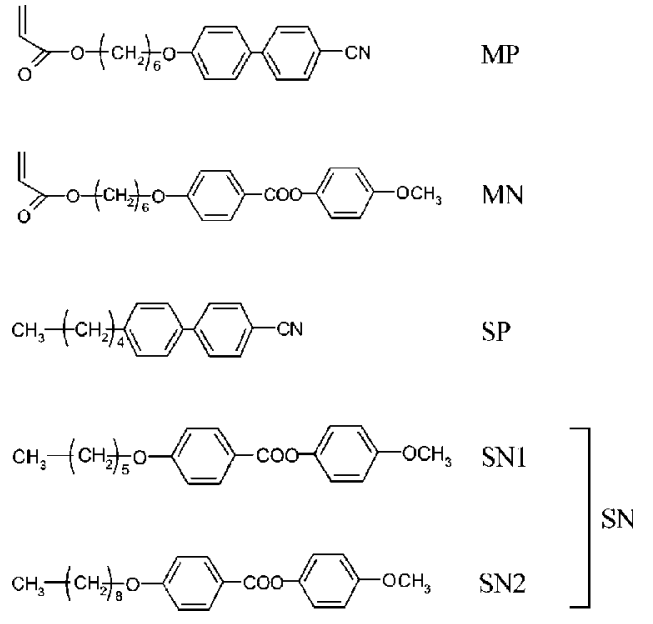

FIG. 1. Molecular structures of employed acrylate mesogenic monomers and nematic solvents. The molar ratio of SN-1 and SN-2 in $\mathrm{SN}$ is $50: 50$.

various mesogen contents and cross-linking densities. The time dependence of the deformation in response to the application and removal of electrical fields is also investigated. The results in the present study offer a basis for the understanding of electrically driven deformations of nematic gels as well as their applications to soft actuators.

\section{EXPERIMENT}

\section{A. Sample preparation}

The side chain nematic networks with positive or negative dielectric anisotropy (designated as LCN-P or LCN-N) were prepared by using the acrylate mesogenic monomer MP or $\mathrm{MN}$, respectively. The molecular structures of MP and MN are shown in Fig. 1, and they were synthesized by the methods in the literature [25,26]. 1,6-hexanediol diacrylate and $2,2^{\prime}$-azobisisobutyronitrile were employed as cross-linker and initiator, respectively. To prepare the nematic networks with various nematicity, the mixtures with different compositions of the monomer MP and the nonmesomorphic styrene monomer were copolymerized. The details of the network preparation were described elsewhere [27]. The nematic networks were made by radical polymerization of the reactant mixtures in capillaries with diameters of several hundreds of micrometers. The cross-linking reaction was carried out at $80{ }^{\circ} \mathrm{C}$ where both MP and MN were in the isotropic phase. The resulting cylindrical gels were separated from the capillaries, and immersed in toluene to wash out the unreacted materials. The swollen gels were gradually deswollen in the mixtures of toluene and methanol with successive compositions, increasing the methanol content stepwise. The fully deswollen gels were completely dried in air. Table I summarizes the characteristics of each network. The numeral $m$ in the sample code LCN-P- $m$ represents the molar fraction of MP in the feed. The cross-linker concentration in the feed for all samples excepting LCN-P-100-H is $1 \mathrm{~mol} \%$ in the total reactants.

The dry networks were allowed to swell in a low molecular mass LC, SP or SN with positive or negative dielectric
TABLE I. Characteristics of the network samples.

\begin{tabular}{lcrr}
\hline \hline \multicolumn{1}{c}{ Network } & $\begin{array}{c}\text { Mesogenic } \\
\text { monomer content }{ }^{\mathrm{a}} \\
{[\mathrm{mol} \%]}\end{array}$ & $\begin{array}{c}\text { Cross-linker } \\
\text { concentration } \\
{[\text { bol \%] }}\end{array}$ & $\begin{array}{r}T_{N I}^{N \mathrm{c}} \\
\left({ }^{\circ} \mathrm{C}\right)\end{array}$ \\
\hline LCN-P-100 & 100 & 1 & 131 \\
LCN-P-100-H & 100 & 10 & 120 \\
LCN-P-95 & 95 & 1 & 122 \\
LCN-P-80 & 80 & 1 & 115 \\
LCN-P-70 & 70 & 1 & 99 \\
LCN-P-50 & 50 & 1 & $\mathrm{~d}$ \\
LCN-N-100 & 100 & 1 & 125 \\
\hline \hline
\end{tabular}

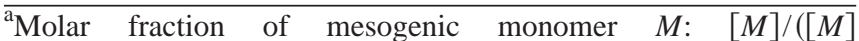
$+[$ styrene monomer $])$.

${ }^{b}$ Molar fraction of the cross-linker in the total reactants.

${ }^{c}$ Nematic-isotropic transition temperature in the dry state evaluated by polarized microscopy.

${ }^{\mathrm{d}}$ No nematicity.

anisotropy, respectively. The solvent SP, conventionally called $5 \mathrm{CB}$, was purchased from Aldrich. The solvent SN was a miscible mixture of $\mathrm{SN}-1$ and $\mathrm{SN}-2$ with the molar ratio 50:50. The molecular structures of $\mathrm{SP}, \mathrm{SN}-1$, and $\mathrm{SN}-2$ are illustrated in Fig. 1. The mixing of SN-1 and SN-2 broadens the temperature range of the nematic phase. Each of $\mathrm{SN}-1$ and $\mathrm{SN}-2$ was synthesized in almost the same manner as the monomer MN. The nematic-isotropic $(N-I)$ transition temperatures of SP and SN were 34.2 and $76.7^{\circ} \mathrm{C}$, respectively. The fully swollen nematic gels showed a single nematic phase composed of the nematic networks and solvents, and no phase separation inside the gels was observed. Table II tabulates the $\mathrm{N}-\mathrm{I}$ transition temperature in the fully swollen state of each network and the degree of equilibrium swelling $(Q)$ at the temperatures in electrical deformation measurements. All networks swelled isotropically even in the nematic phase owing to the polydomain texture with a finite orientational order in the individual domains but without global orientation of the director. Thus the swelling degree $Q$, defined as the ratio of the volumes in the dry and fully swollen states, was evaluated from the cube of the ratio of the gel diameters.

\section{B. Measurements of electrically induced deformation}

The deformation of the gels under dc fields was observed by optical microscopy as schematically shown in Fig. 2 . The swollen cylindrical gels with diameter of ca. $1 \mathrm{~mm}$ were placed without mechanical constraint between Pt electrodes separated by $2 \mathrm{~mm}$, and the cell was filled with the nematic solvent. The surface level of the solvent was adjusted to be low enough so that the outline of the gel was distinctly visible when viewed through the microscope (but high enough to immerse the gel completely). The temperature of the cell was controlled by a hot stage Mettler FP-82. The swelling was equilibrated at each temperature before applying electric fields.

The dimensions of the gels $d_{i}(i=x, y)$ parallel and normal to the field direction were measured as a function of the 
TABLE II. Nematic-isotropic transition temperatures of nematic gels and swelling degrees at the temperatures in electrical deformation measurements.

\begin{tabular}{ccccc}
\hline \hline Network/solvent & $\begin{array}{c}T_{N I}^{G \text { a }}\left({ }^{\circ} \mathrm{C}\right) \\
\text { LCN-P-100/SP }\end{array}$ & $\begin{array}{c}\text { Phase } \\
(\text { gel/surrounding solvent })^{\mathrm{b}}\end{array}$ & $\begin{array}{c}\text { Temperature } \\
\left({ }^{\circ} \mathrm{C}\right)\end{array}$ & $Q^{\mathrm{c}}$ \\
\hline & 50.3 & $N / N$ & 25 & 15 \\
& & $N / I$ & 38 & 4.5 \\
LCN-P-100/SN & 102 & $I / I$ & 60 & 17 \\
LCN-N-100/SN & 85.8 & $N / I$ & 81 & 2.6 \\
& & $N / I$ & 81 & 8.9 \\
LCN-N-100/SP & 45.8 & $I / I$ & 100 & 33 \\
LCN-P-100-H/SP & 64.2 & $N / N$ & 25 & 29 \\
LCN-P-95/SP & 49.2 & $N / N$ & 25 & 2.7 \\
LCN-P-80/SP & 48.2 & $N / N$ & 25 & 7.1 \\
LCN-P-70/SP & 41.2 & $N / N$ & 25 & 7.8 \\
LCN-P-50/SP & 34.2 & $N / N$ & 25 & 7.9 \\
\hline \hline
\end{tabular}

${ }^{a}$ Nematic-isotropic transition temperature in the fully swollen state evaluated by polarized microscopy.

${ }^{\mathrm{b}} N$, nematic phase; $I$, isotropic phase.

${ }^{c}$ Volume ratio in the fully swollen and dry states.

strength of electric field $(E)$. As shown in Fig. 2, the field direction coincides with the $x$ axis in the orthogonal coordinates. The dimension in the $z$ direction was not directly measured, but $d_{z}$ was evaluated from the volume constancy before and after deformation, i.e., $d_{x} d_{y} d_{z}=d_{x 0} d_{y 0}{ }^{2}$ where the subscript 0 denotes the undeformed state. As demonstrated later, the volume of the gels under electric fields does not noticeably change even after long times. The voltage was stepwise increased, and the dimensions $d_{x}$ and $d_{y}$ at each $E$ were measured, ca. $30 \mathrm{~s}$ after the application of the corresponding voltage. This time was long enough to achieve the deformation in the steady state under the voltage concerned. The time dependence of the deformation in response to the application and removal of electric fields was also examined in a separate experiment. The principal strain $\gamma_{i}(i=x, y, z)$ in each direction is evaluated by

$$
\gamma_{i}=\left(d_{i}-d_{i 0}\right) / d_{i 0} .
$$

In the geometry employed, the field strength effectively acting on the gel $\left(E_{g}\right)$ is related to that between the elec-

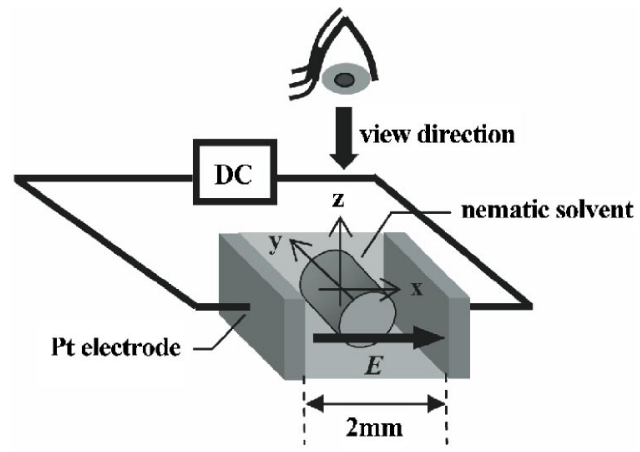

FIG. 2. Schematics of the measurement for the electrical deformation of nematic gels. trodes (E) separated by the distance $d_{t}$ as $E_{g}=E d_{t} /\left\{d_{x}\right.$ $\left.+\left(\varepsilon_{g} / \varepsilon_{s}\right) d_{s}\right\}$ where $\varepsilon_{g}$ and $\varepsilon_{s}$ are the dielectric constants of the gel and surrounding solvent along the field axis, respectively, and $d_{s}\left(=d_{t}-d_{x}\right)$ is the length occupied by the surrounding solvent. The high solvent contents of all gels (except for LCN-P-100-H), over $85 \mathrm{vol} \%$, may allow us to assume $\varepsilon_{g} \approx \varepsilon_{s}$ (yielding $E_{g} \approx E$ ), but the difference in orientation between the nematogens inside and outside the gel markedly complicates the accurate estimation of $E_{g}$ [28]. Thus we employ the apparent field strength $E$ for the discussion hereafter.

\section{RESULTS AND DISCUSSION}

\section{A. Electrical deformations of LCN-P-100/SP}

Figure 3 shows the optical micrographs for LCN-P-100$1 / \mathrm{SP}$ at $25^{\circ} \mathrm{C}$ in the totally nematic phase before and after applying the electric field of $E=0.6 \mathrm{MV} / \mathrm{m}$. The gel is stretched in the field direction and compressed in the direction normal to the field axis. Figure 4(a) displays $\gamma_{i}$ ( $i$ $=x, y, z)$ as a function of $E$ for LCN-P-100-1/SP at $25^{\circ} \mathrm{C}$ in the totally nematic $(N / N)$ phase, and $38^{\circ} \mathrm{C}$ in the $N / I$ phase where the gel is nematic whereas the surrounding solvent is isotropic. Hereafter we designate the phases inside and outside the gel as $N($ gel) $/ I$ (surrounding solvent) where $N$ and $I$ denote the nematic and isotropic phases, respectively. At both temperatures, the gel is elongated in the field direction $\left(\gamma_{x}>0\right)$, and laterally compressed along the $y$ and $z$ axes in the same way due to the incompressibility $\left(\gamma_{y} \approx \gamma_{z}<0\right)$, although the data of $\gamma_{i}(i=y, z)$ in the $N / I$ phase were not displayed in the figure. The stretching direction coincides with the director axis in the electrical orientation of the constituent nematogens MP and SP with positive $\Delta \varepsilon$. The degree of deformation grows with increasing $E$, and becomes almost constant at the high field strengths of $E>0.6 \mathrm{MV} / \mathrm{m}$. LCN- 

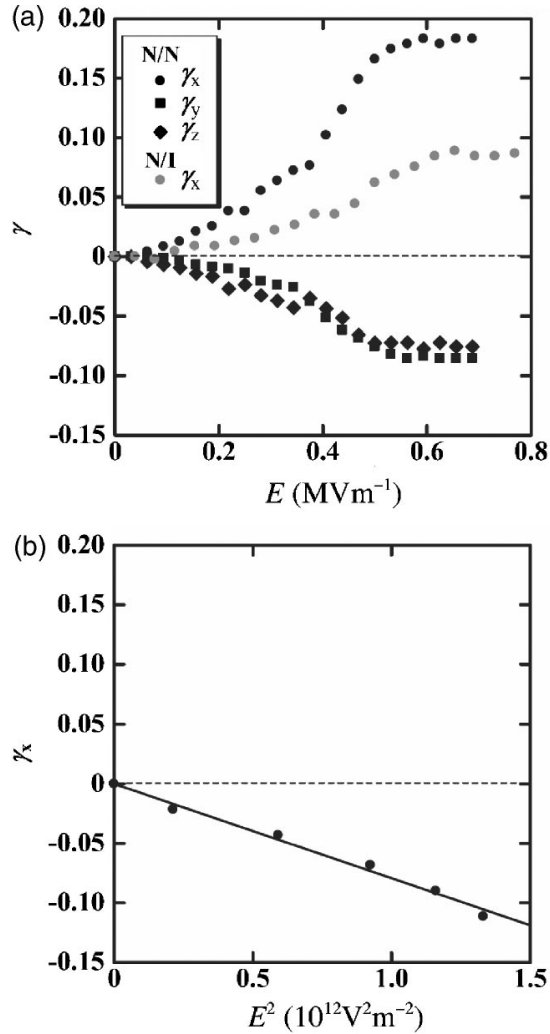

FIG. 3. Principal strains of LCN-P-100/SP (a) in the $N / N$ $(Q=15)$ and $N / I$ phases $(Q=4.5)$ as a function of field strength $(E)$ and (b) in the $I / I$ phase $(Q=17)$ as a function of $E^{2}$.

$\mathrm{P}-100 / \mathrm{SP}$ in the $N / N$ phase exhibits the largest electrical strain (ca. 20\%) among the samples examined. The maximum strain in the $N / I$ phase (ca. $10 \%$ ) is lower than that in the $N / N$ phase [29], primarily due to the smaller swelling degree yielding the higher elastic modulus: The ratio of the maximum strains at the two temperatures (ca. 2) is not far from the elastic compliance ratio expected from the classical rubber elasticity theory [30], $\left(Q_{N / N} / Q_{N / I}\right)^{1 / 3} \approx 1.5$. This suggests that the phase of the surrounding solvent influences the swelling degree (elastic modulus) but does not alter the essential characteristics of electrical deformations such as the stretching direction. A threshold of $E$ (around $0.1 \mathrm{MV} / \mathrm{m}$ ) to yield a finite deformation in the $N / N$ or $N / I$ phase is present, the details of which will be discussed later.

As shown in Fig. 4(b), the gel at $60{ }^{\circ} \mathrm{C}$ in the totally isotropic $(I / I)$ phase exhibits compression in the field direction $\left(\gamma_{x}<0\right)$ in contrast to the stretching $\left(\gamma_{x}>0\right)$ in the nematic phase. The compressive strain linearly depends on $E^{2}$ in accordance with the familiar electrostriction, indicating that the gel in the isotropic phase behaves like the usual paraelectric materials. This result also demonstrates that the electrical deformation dominated by the nematogen alignment needs nematic order as well as large dielectric anisotropy. The shear modulus of the gel in the isotropic phase $\left(G_{i}\right)$ is estimated to be $1.0 \times 10^{3} \mathrm{~Pa}$ from the electrostrictive coefficient $a$ ( $a$ is defined by $\left.\gamma_{x}=-a E^{2}\right)$ on the basis of the relation for paraelectric materials $[21,22,31]$ : (a)
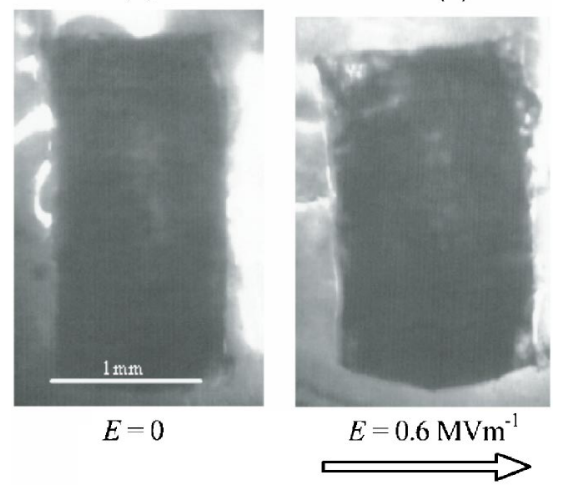

FIG. 4. Optical micrographs of LCN-P-100/SP (a) before and (b) after applying the electric field of $E=0.6 \mathrm{MV} / \mathrm{m}$.

$$
a=\varepsilon_{0}(\varepsilon-1)^{2} /\left(9 G_{i}\right)
$$

where $\varepsilon$ and $\varepsilon_{0}$ are the dielectric constants of materials and vacuum. In the calculation, the literature value of the dielectric constant of the solvent SP in the isotropic phase was used as $\varepsilon$ because of the high solvent content in the gel (94 vol \%) as well as the similar chemical structures of MP and SP. The modulus of the gel in the nematic phase is unknown but will be on the same order of magnitude as that in the isotropic phase, because the modulus of the dry polydomain nematic networks is not significantly influenced by the $N-I$ transition [32], and the swelling degree in the $N / N$ phase is comparable to that in the $I / I$ phase.

\section{B. Electrical deformations of LCN-N-100/SN}

Figure 5(a) illustrates the $E$ dependence of $\gamma_{i}(i=x, y, z)$ for LCN-N-100/SN at $81{ }^{\circ} \mathrm{C}$ in the $N / I$ phase. Measurement in the $N / N$ phase was not possible; the outline of the gel was invisible because there was almost no optical contrast between the swollen gel and the surrounding solvent. As shown before, the phase of the surrounding solvent alters the swelling degree but does not influence the orientation direction of the gel under electric fields. LCN-N-100/SN in the $N / I$ phase is compressed in the field direction $\left(\gamma_{x}<0\right)$, in other words, stretched normal to the field direction $\left(\gamma_{y} \approx \gamma_{z}>0\right)$. The stretching directions agree with the director axes in the electrical alignment of the constituent nematogens $\mathrm{MN}$ and $\mathrm{SN}$ with negative $\Delta \gamma$ : The director of the dielectrically negative nematogens is electrically oriented not in a unique direction but in the plane (including $y$ and $z$ directions) normal to the field axis. The compressive strains $\gamma_{x}$ are almost constant in the region of $E>0.5 \mathrm{MV} / \mathrm{m}$. As seen in Fig. 5(b), $\gamma_{x}$ in the $I / I$ phase is also compressive and in proportion to $E^{2}$. The compression along the field axis is different in major origin between the nematic and isotropic phases: The former is mainly driven by the electrical orientation of the nematogens, while the latter is purely caused by electrostriction. The plateau value of $\gamma_{x}$ in the $N / I$ phase (ca. 7\%) is rather higher than $\gamma_{x}$ (less than 1\%) in the $I / I$ phase at the corresponding $E$, despite the much smaller swelling degree of the nematic gel $\left(Q_{N / I} \approx Q_{I / I} / 4\right)$, suggesting the large effect of the nematogen alignment on the deformation. 

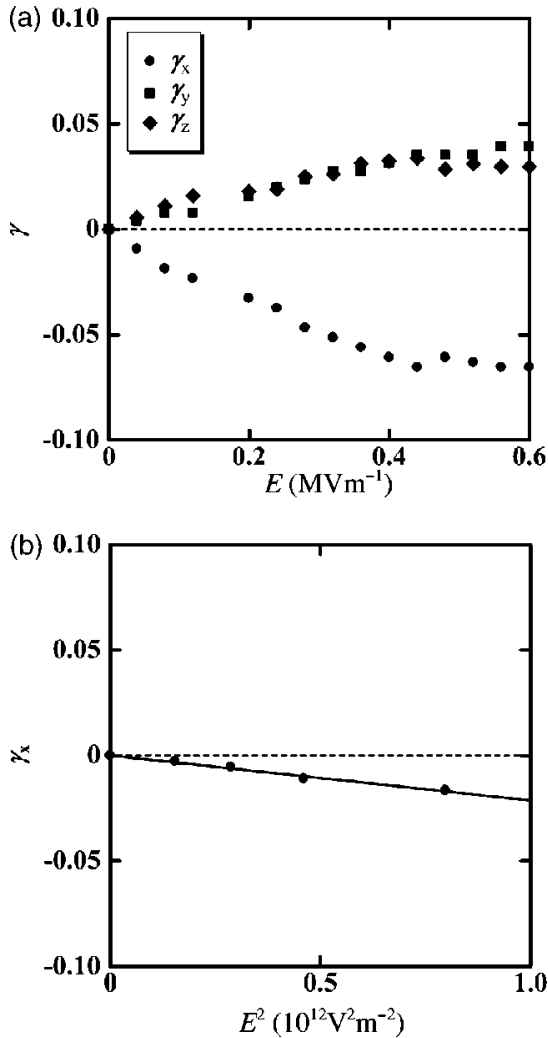

FIG. 5. Principal strains of LCN-N-100/SN (a) in the $N / I$ phase $(Q=8.9)$ as a function of field strength $(E)$ and (b) in the $I / I$ phase $(Q=33)$ as a function of $E^{2}$.

\section{Electrical deformations of LCN-P-100/SN and $\mathrm{LCN}-\mathrm{N}-100 / \mathrm{SP}$}

Figure 6 displays the $E$ dependence of $\gamma_{x}$ for LCN-P$100 / \mathrm{SN}$ and LCN-N-100/SP, i.e., the combinations of networks and solvents with opposite signs of $\Delta \varepsilon$. The former system is examined in the $N / I$ phase because of insufficient optical contrast between the gel and surrounding solvent in the $N / N$ phase, while the latter system is studied in the $N / N$ phase. Of importance is that both systems show compressive strains in the field direction. It should be noticed that the deformation of LCN-N-100/SP is not governed by the positive dielectric anisotropy (i.e., not elongated in the field direction), despite the markedly high content of the solvent (97 vol \%) with a positive large $\Delta \varepsilon$. According to the results in Figs. 3-6 only the system of the network and solvent both of which have positive $\Delta \varepsilon$ (LCN-P-100/SP) is stretched in the field direction whereas all other three systems are compressed. This indicates that combinations of the network and solvent with identical signs of $\Delta \varepsilon$ are needed for the electrical deformation dominated by the nematogen orientation effect. When the signs of $\Delta \varepsilon$ of the components are opposite, the effect of mesogen alignment on the deformation is much reduced or diminished because of the discord in the director directions of the constituent nematogens. As a result, the electrostrictive effect becomes dominant yielding a compression along the field axis. However, the resulting strain is not linearly dependent on $E^{2}$, implying that there exists a finite contribution of the mesogen orientation effect and/or the ef-

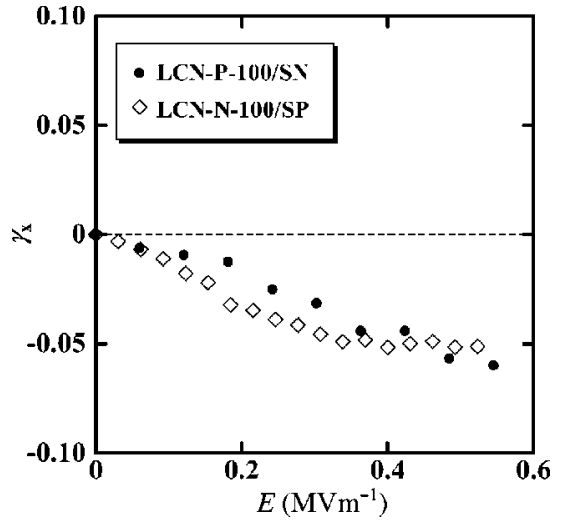

FIG. 6. Principal strain along the field direction for LCN-P$100 / \mathrm{SN}$ in the $N / I$ phase $(Q=2.6)$ and $\mathrm{LCN}-\mathrm{N}-100 / \mathrm{SP}$ in the $N / N$ phase $(Q=25)$ as a function of field strength $(E)$.

fect of a strong dipole interaction between the nematic domains on electrostriction.

LCN-N-100/SN in the nematic phase is expected to be elastically rather harder than LCN-N-100/SP on the basis of the considerably smaller $Q$. Nevertheless, the values of $\gamma_{x}$ at high $E$ in the two systems [Figs. 5(a) and 6] are comparable. This also reveals a significant contribution of the coupling of the network and solvent with identical signs of $\Delta \varepsilon$ to the electrical deformation.

All nematic gels examined here exhibited no appreciable change in birefringence before and after deformations, indicating that the electric fields do not induce a definite orientation of the polydomain structure. The electric fields cause a finite orientation of the individual domains yielding the macroscopic deformation observed, but the induced variation in local orientation is so small that the change in global birefringence may be masked due to a relatively large sample thickness (ca. $1 \mathrm{~mm})$. It is likely that the saturation of the strain at high fields reflects the limitation of the electrical orientation of the polydomain structure. The electrical deformation of monodomain nematic gels with global director orientation is an interesting issue, and it will be investigated in a separate study [33].

\section{Effect of cross-linking density on electrical deformations}

Figure 7 shows the comparison of $\gamma_{x}$ for the swollen networks LCN-P-100/SP with different cross-linking densities in the $N / N$ phase. The swelling degree of LCN-P-100-H is considerably lower than that of LCN-P-100 due to the higher elastic modulus. The network with the higher cross-linking density shows less maximum deformability (ca. 5\%), and needs a higher $E$ (ca. $1.4 \mathrm{MV} / \mathrm{m}$ ) to reach the maximum deformation. These are attributed to two effects of high cross-linking density: One is to enhance the elastic modulus of swollen networks; the other is to reduce the spatial freedom for the pendant mesogens, which hinders the growth of the orientation.

\section{E. Effect of network nematicity on electrical deformations}

Figure 8 depicts the $E$ dependence of $\gamma_{x}$ for the LCN-P$m / \mathrm{SP}$ with various molar fractions of $\mathrm{MP}(m)$ in the $N / N$ 


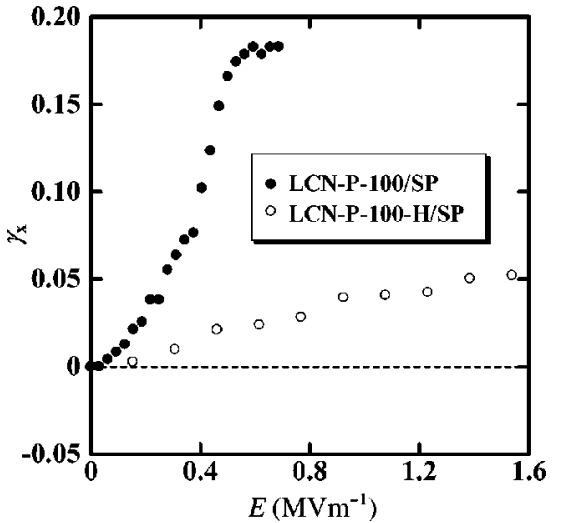

FIG. 7. Principal strain along the field direction for LCN-P$100 / \mathrm{SP}(Q=15)$ and LCN-P-100-H/SP $(Q=2.7)$ in the $N / N$ phase as a function of field strength $(E)$.

phase. As can be seen in Table I, a decrease in $m$ yields a reduction in $T_{N}^{N}$, i.e., a reduction in network nematicity. A decrease in network nematicity gives rise to two pronounced effects on the electric deformation: The first is to increase the threshold field strength $\left(E_{c}\right)$ to yield a finite deformation; the second is to lessen the tensile strain in the field direction. Of interest is that the deformation along the field axis exhibits a crossover from stretching $\left(\gamma_{x}>0\right)$ to compression $\left(\gamma_{x}<0\right)$ around $m=0.5$. The networks with $m<0.4$ showed no appreciable electrical distortion due to the low degrees of swelling $(Q<5)$ as well as the low MP content.

The presence of $E_{c}$ for LCN-P/SP totally composed of dielectrically positive nematogens stems from the cancellation of the two strains in the opposite directions: the tensile strain induced by the nematogen alignment, and the compressive strain originating from electrostriction. In contrast, as is evident in Figs. 5 and 6, such a threshold field strength is absent for LCN-N/SN, LCN-P/SN, and LCN-N/SP all of which contain dielectrically negative nematogens. For LCN$\mathrm{P} / \mathrm{SP}$, the extensive strain driven by the mesogen alignment becomes smaller with decreasing $m$ owing to a reduction in network nematicity. Meanwhile, the compressive strain caused by electrostriction will be almost unaltered by $m$ : The

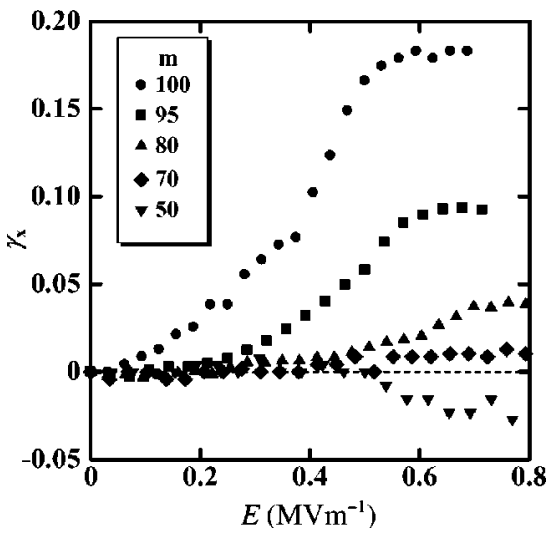

FIG. 8. Principal strain along the field direction for LCN-P$\mathrm{m} / \mathrm{SP}$ with different MP content $(\mathrm{m})$ in the $N / N$ phase as a function of field strength $(E)$.

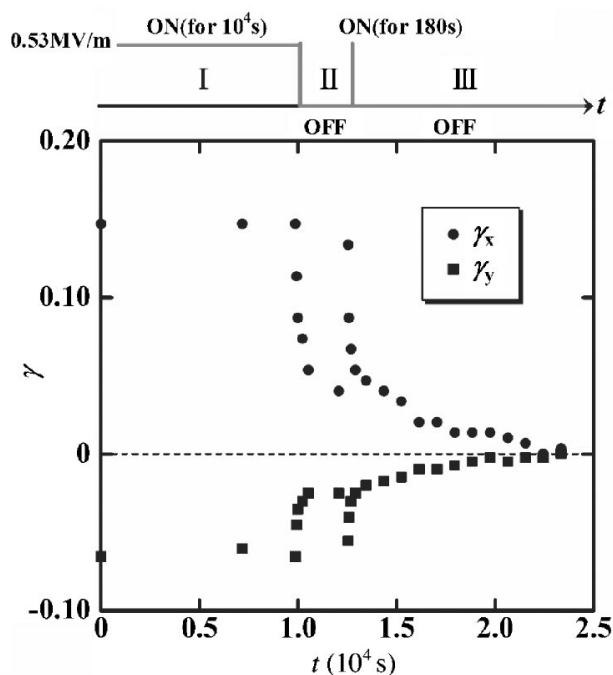

FIG. 9. Time dependence of the strains for LCN-P-100/SP in the $N / N$ phase in response to the application and removal of the electric field of $E=0.53 \mathrm{MV} / \mathrm{m}$. The protocol for the electric field is indicated in the figure.

swelling degrees of the networks with $m>0.5$ are high and almost independent of $m$, implying that neither the dielectric constant nor the elastic modulus governing the electrostiction significantly differs among LCN-P/SP with $m>0.5$. As a consequence, the $E$ region showing almost zero strain is apparently broadened with decreasing $m$. When the network nematicity becomes quite small, the compressive strain by electrostriction in the high $E$ region exceeds the extensive strain by the weak effect of nematogen orientation. This explains the crossover from $\gamma_{x}>0$ (elongation) to $\gamma_{x}<0$ (compression) around $m=0.5$ observed.

The results in Fig. 8 show the presence of the electrostrictive effect for LCN-P/SP in the nematic phase. On the basis of this result, it is expected that strains at high $E$ do not saturate in LCN-N/SN where the nematogen alignment yields compressive strain in the same direction as the electrostriction. However, the experimental data exhibit a plateau in strain at high $E$ in disagreement with this expectation, which may be because the electrostrictive effect in LCN$\mathrm{N} / \mathrm{SN}$ is too small to appear definitely in the $E$ range examined. For instance, the electrostriction for LCN-N/SN in the isotropic phase [Fig. 5(b)] is much smaller than that for LCN-P/SP [Fig. 4(b)] probably due to the rather smaller dielectric constant.

\section{F. Time dependence of electrical deformations}

Figure 9 illustrates the time dependence of $\gamma_{x}$ and $\gamma_{y}$ for LCN-P-100/SP in response to the electric fields according to the protocol shown in the figure. Upon the application of the electric field, the induced deformation reaches the steady state within a second. Field application for ca. $3 \mathrm{~h}$ (region I) yields no noticeable change in $\gamma$, indicating that the swelling degree is almost unaltered by the electric fields. After the removal of the electric field at $t=1.0 \times 10^{4} \mathrm{~s}$, the gel exhibits shape recovery but perfect shape recovery requires a finite 


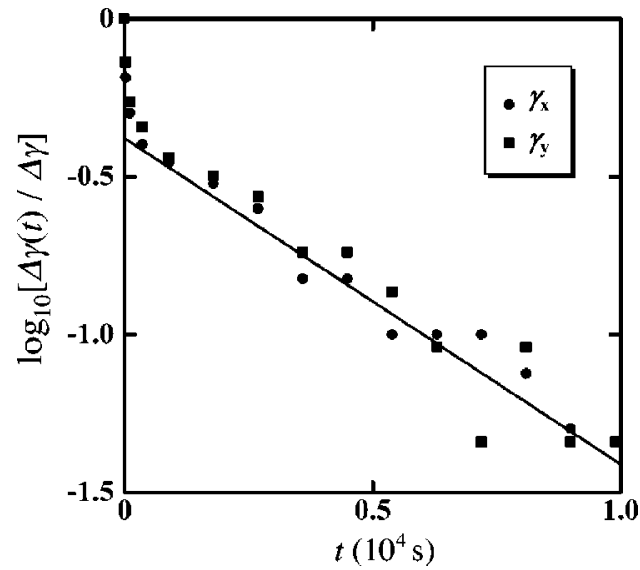

FIG. 10. Semilog plots of the reduced strains $\Delta \gamma(t) / \Delta \gamma$ vs time for LCN-P-100/SP in the $N / N$ phase after the removal of the electric field: $\Delta \gamma(t)=\gamma(t)-\gamma(\infty), \Delta \gamma=\gamma(0)-\gamma(\infty)$, where $\gamma(0)$ and $\gamma(\infty)$ are the strains just after the field application and in the undeformed state (or in the long time limit), respectively; $t=0$ in the figure corresponds to the time at the field removal. The data in region III in Fig. 9 were employed. The slope of the line yields the longest characteristic time of $4.0 \times 10^{3} \mathrm{~s}$ for shape recovery.

time. When an electric field with identical strength is applied again halfway in the recovery process at $t \approx 1.3 \times 10^{4} \mathrm{~s}$, the gel is elongated again up to almost the same degree as $\gamma_{x}$ upon the first field application. Region III corresponds to the whole process of shape recovery after the removal of the electric field. About $9 \times 10^{3} \mathrm{~s}$ is needed to recover the original shape perfectly.

Figure 10 displays a semilog plot of $\Delta \gamma(t) / \Delta \gamma$ for the data in region III where $\Delta \gamma(t)=\gamma(t)-\gamma(\infty), \Delta \gamma=\gamma(0)-\gamma(\infty)$ : $\gamma(0)$ and $\gamma(\infty)$ are the strains just after the field application and in the undeformed state (or in the long time limit), respectively; $t=0$ in Fig. 10 corresponds to the time at field removal. No significant difference in the relaxation process between $\gamma_{x}$ and $\gamma_{y}$ is observed, indicating that there exists no anisotropy in the shape recovery process. The data at relatively long times of $t>900 \mathrm{~s}$ fall on the straight line. The longest relaxation time $\tau$ for shape recovery is evaluated to be $4.0 \times 10^{3} \mathrm{~s}$ from the slope of the line. The upward deviation of the data at short times from the straight line suggests that faster relaxation modes are present, and that the whole process can be described by a multiexponential relaxation function. The slow process of shape recovery probably re- flects structural relaxation in the polydomain nematic texture from the oriented state to the random state.

\section{CONCLUSIONS}

The nematic networks swollen by nematic solvents under electric fields are stretched in the director direction of electrically induced alignment of the nematogens, when the signs of $\Delta \varepsilon$ for the network and solvent are identical: The dielectrically positive (negative) nematic gels are elongated (compressed) along the field axis. The nematogen alignment effect yields a maximum strain of ca. $20 \%$ at $E \approx 0.5 \mathrm{MV} / \mathrm{m}$ among the samples examined. In the case where the signs of $\Delta \varepsilon$ for the constituent nematogens are opposite, the strain in the field direction is compressive because the effect of nematogen orientation becomes too small to exceed the electrostrictive effect due to the discord in the director directions of the constituent nematogens. In the isotropic phase, all gels show compressive strain along the field axis in proportion to $E^{2}$ purely originating from electrostriction. These results indicate that electrical deformation governed by the nematogen alignment effect requires nematic order as well as large $\Delta \varepsilon$ with identical signs.

In a system of network and solvent both of which have positive $\Delta \varepsilon$, a threshold value of $E$ to yield a finite deformation is present in the low $E$ region as a result of the cancellation of the two strains in the opposite directions: the extensive strain stemming from the nematogen alignment effect, and the compressive strain originating from electrostriction. As the network nematicity gets smaller, $E_{c}$ becomes larger and the tensile strain along the field axis decreases because of a reduction in the mesogen orientation effect.

The application of an electric field quickly deforms the nematic gels, while perfect shape recovery after the removal of the field needs a finite time in the order of $10^{3} \mathrm{~s}$. The slow process of shape recovery presumably results from structural relaxation in the polydomain nematic texture from the oriented to the random state.

\section{ACKNOWLEDGMENTS}

Y.O.A. thanks the Japan Society for the Promotion of Science (JSPS) for partial support (Grant No. 15005225). Further support was provided by a Grant-in-Aid (No. 16750186) and the 21st century COE program "COE for a United Approach to New Materials Science" from the Ministry of Education, Culture, Sports, Science, and Technology, Japan.
[1] H. Finkelmann and H. R. Brand, Trends Polym. Sci. 2, 222 (1994).

[2] M. Warner and E. M. Terentjev, Liquid Crystal Elastomers (Oxford University Press, Oxford, 2003).

[3] A. R. Tajbackhsh and E. M. Terentjev, Eur. Phys. J. E 6, 181 (2001)

[4] H. Finkelmann, A. Grave, and M. Warner, Eur. Phys. J. E 5, 281 (2001).
[5] D. L. Thomsen, P. Keller, J. Nacri, R. Pink, H. Jeon, D. Shenoy, and B. R. Ratna, Macromolecules 34, 5868 (2001).

[6] K. Urayama, Y. Okuno, T. Kawamura, and S. Kohjiya, Macromolecules 35, 4567 (2002).

[7] K. Urayama, Y. Okuno, and S. Kohjiya, Macromolecules 36, 229 (2003).

[8] K. Urayama, Y. Okuno, T. Nakao, and S. Kohjiya, J. Chem. Phys. 118, 2903 (2003). 
[9] Y. Okuno, K. Urayama, and S. Kohjiya, J. Chem. Phys. 118, 9854 (2003).

[10] K. Urayama, Y. O. Arai, and T. Takigawa, Macromolecules 37, 6161 (2004).

[11] P. Calvert, in Electroactive Polymer (EAP) Actuators as Artificial Muscles-Reality, Potential, and Challenges, edited by Y. Bar-Cohen (SPIE, Bellingham, WA, 2001).

[12] R. Zentel, Liq. Cryst. 1, 589 (1986).

[13] N. R. Barnes, F. J. Davis, and G. R. Mitchell, Mol. Cryst. Liq. Cryst. 168, 13 (1989).

[14] E. M. Terentjev, M. Warner, and H. Bladon, J. Phys. II 4, 667 (1994).

[15] R. Kishi, H. Suzuki, H. Ichijo, and O. Hirasa, Chem. Lett. 1994, 2257.

[16] R. Kishi, T. Kitano, and H. Ichijo, Mol. Cryst. Liq. Cryst. Sci. Technol., Sect. A 280, 109 (1996).

[17] C. Huang, Q. Zhang, and A. Jákli, Adv. Funct. Mater. 13, 525 (2003).

[18] W. Lehmann, H. Skupin, C. Tolksdorf, E. Gebhard, R. Zentel, P. Krüger, M. Lösche, and F. Kremer, Nature (London) 410 , 447 (2001).

[19] S. Courty, J. Mine, A. R. Tajbakhsh, and E. M. Terentjev, Europhys. Lett. 64, 654 (2003).

[20] C. Kittel, Introduction to Solid State Physics, 8th ed. (John Wiley \& Sons, Chichester, 2004).

[21] H. J. Winkelhahn, H. H. Winter, and D. Neher, Appl. Phys. Lett. 64, 1347 (1994).

[22] H. J. Winkelhahn, T. Pakula, and D. Neher, Macromolecules 29, 6865 (1996).

[23] P. G. de Gennes and J. Prost, Physics of Liquid Crystals (Ox- ford University Press, Oxford, 1994).

[24] S. Chandrasekhar, Liquid Crystals, 2nd ed. (Cambridge University Press, Cambridge, U.K., 1992).

[25] V. P. Shivaev, S. G. Kostromin, and N. A. Plate, Eur. Polym. J. 18, 651 (1982).

[26] M. Portugall, H. Ringsdorf, and R. Zentel, Makromol. Chem. 183, 2311 (1982).

[27] Y. O. Arai, K. Urayama, and S. Kohjiya, Polymer 45, 5127 (2004).

[28] The electrically induced alignment of the surrounding solvent (the low molecular mass LC) is fully achieved in the whole $E$ range examined. Meanwhile, we have no information about the induced orientation of the nematogens inside the gels, which changes with $E$. The gels show no noticeable variation in birefringence before and after applying electric fields.

[29] The difference in $\varepsilon$ of the surrounding solvent between the isotropic and nematic phases will affect $E_{g}$. This may influence the shape of the $\gamma-E$ curve but does not alter the maximum strain.

[30] L. R. G. Treloar, The Physics of Rubber Elasticity, 3rd ed. (Oxford University Press, Oxford, 1975).

[31] In the expression for $a$ in Refs. [21,22], the contribution of the Maxwell force between the electrodes is considered together with the electrostrictive effect. In Eq. (2), the term regarding the Maxwell force is absent because the electrodes are fixed in the present geometry.

[32] T. Pakula and R. Zentel, Makromol. Chem. 192, 2401 (1991).

[33] K. Urayama, S. Honda, and T. Takigawa, Macromolecules 38, 3574 (2005). 